\title{
eJRIEPS
}

Ejournal de la recherche sur l'intervention en éducation physique et sport

15 | 2008

Varia

\section{L'identification des savoirs mobilisés par le professeur d'EPS en situation : le cas du franchissement par redressement au saut de cheval}

Fabienne Brière-Guenoun

\section{(2) OpenEdition}

1 Journals

Édition électronique

URL : https://journals.openedition.org/ejrieps/5833

DOI : 10.4000/ejrieps.5833

ISSN : 2105-0821

Éditeur

ELLIADD

Référence électronique

Fabienne Brière-Guenoun, «L'identification des savoirs mobilisés par le professeur d'EPS en situation : le cas du franchissement par redressement au saut de cheval », eJRIEPS [En ligne], 15 | 2008, mis en ligne le 01 juillet 2008, consulté le 03 mai 2021. URL : http://journals.openedition.org/ejrieps/5833 ; DOI : https://doi.org/10.4000/ejrieps.5833

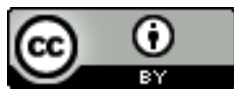

La revue eJRIEPS est mise à disposition selon les termes de la Creative Commons Attribution 4.0 International License. 
L'identification des savoirs mobilisés par le professeur d'EPS en situation : le cas du franchissement par redressement au saut de cheval.

\section{Fabienne Brière-Guenoun}

Laboratoire DiDiST-CREFI-T, Université Paul Sabatier, Toulouse III, France UFRSTAPS d'Orléans.

\section{Résumé}

Cette recherche concerne la nature, les fonctions et les processus d'activation des savoirs mobilisés par le professeur d'EPS. Elle repose sur l'observation d'un enseignant proposant l'étude du franchissement par redressement au saut de cheval, lors d'un cycle ordinaire de gymnastique, en classe de quatrième au collège. Les savoirs de l'enseignant sont révélés par ses gestes, qui s'expriment dans des "tâches de conception et d'organisation de dispositifs d'étude » et des «tâches d'aide à l'étude » (Chevallard, 1999). En référence à la théorie anthropologique du didactique (Chevallard, 1997), ces tâches d'enseignement dépendent d'organisations complexes, nommées "praxéologies 》, constituées d'un bloc pratique (le système tâche-technique) et d'un bloc théorique (le système technologico-théorique). Les quatre premières leçons du cycle font l'objet d'enregistrements filmés suivis d'entretiens de type " auto-confrontations ». Des entretiens de type semi-directif, en début et en fin de cycle, ont aussi été réalisés. Les résultats montrent que les savoirs exploités par le professeur appartiennent à la fois aux registres “savants» et "experts» (Johsua, 1996). Ils révèlent l'influence de ses diverses appartenances institutionnelles ainsi que ses convictions personnelles.

Les enjeux d'une formation au métier de professeur d'EPS appellent, selon nous, une réelle réflexion sur les mécanismes expliquant la construction de connaissances utiles et utilisables pour le professeur. La question des savoirs professionnels des enseignants entre dans une problématique récurrente en sciences de l'éducation et ne peut être envisagée indépendamment des contraintes de la professionnalisation. Les différents travaux portant sur les savoirs et connaissances professionnels mettent en évidence leur dimension plurielle et multi-dimensionnelle, souvent ancrée sur des distinctions dichotomiques du type «savoirs pratiques et savoirs théoriques » (Barbier, 1996; 
Gauthier et al. 1997), leur dimension très contextualisée qui renvoie à leur caractère tacite (Poliany, 1967) et leur forte incorporation dans les pratiques (Amade-Escot, 1998). C'est en raison de leur dimension située que nous avons envisagé d'étudier les savoirs de l'enseignant d'un point de vue didactique, en prenant particulièrement en compte les contenus enseignés en éducation physique et sportive à partir d'une observation des pratiques effectives. Cette recherche s'inscrit dans le champ de la didactique, dont elle retient la nécessité de considérer, de façon concomitante, les trois instances du système didactique (l'élève, l'enseignant, le savoir) en contexte d'enseignement naturel (AmadeEscot, 2003 ; Mercier, Schubauer-Léoni \& Sensevy, 2002). Aussi, même si notre étude a pour objet les savoirs du professeur, l'action de l'enseignant est interprétée en regard des actions des élèves et des incidences sur le savoir en jeu au sein de la classe. La recherche présentée vise à repérer la nature, la structuration, les fonctions et les modes d'activation des savoirs chez un professeur d'EPS en situation d'enseignement. Elle s'intéresse plus précisément aux façons dont il met effectivement en scène le thème d'étude «franchir par redressement au saut de cheval», au cours d'un cycle de gymnastique au collège.

\section{Cadre théorique}

Les savoirs de l'enseignant sont appréhendés selon le point de vue de la théorie anthropologique du didactique (Chevallard, 1992, 2002), qui postule l'importance du contexte institutionnel dans la compréhension des phénomènes transpositifs. Selon cette approche, les processus d'appropriation et d'enseignement sont dépendants des « rapports au savoir » qui se nouent au sein des institutions les abritant, et dont une part seulement est rendue publique aux yeux de cette institution. Les savoirs du professeur sont ici étudiés à partir de ses «gestes », qui s'expriment dans des tâches de « conception et d'organisation de dispositifs d'étude » et dans des «tâches d'aide à l'étude » (Chevallard, 1997). Ces gestes professionnels dépendent eux-mêmes d'organisations complexes, nommées «praxéologies » mettant en relation deux systèmes : un système tâches-techniques et un système théorico-technologique. A l'articulation de ces deux systèmes, la théorie justifie, explique et produit la (ou les) technique(s) et justifie, explique et produit la (ou les) technologie(s). La notion de praxéologie favorise ainsi une description fine des pratiques (le système « tâches-techniques), en les rattachant à ce qui les justifie au plan théorique (le système «théorico-technologique »). Par ailleurs, toute activité d'enseignement met en jeu deux types de praxéologies : a) des praxéologies disciplinaires (gymniques dans notre étude) qui renvoient à la réalité disciplinaire pouvant 
se construire dans une classe; b) des praxéologies didactiques qui concernent les manières dont se réalise l'étude de ce thème (Chevallard, 1999).

C'est parce que la notion de praxéologie désigne une organisation bi-polaire reliant le logos à la praxis, qu'elle nous a paru pertinente pour appréhender les savoirs de l'enseignant, qui, rappelons le, sont encapsulés dans l'action et peu dissociables des pratiques. Dans notre étude, les praxéologies sont révélées par les façons dont le professeur réalise la communication des objets de savoir gymniques, lors de la conception et la régulation des dispositifs d'apprentissage dans les interactions didactiques, au cours des différents moments structurant cet enseignement. Les savoirs de l'enseignant sont alors inférés à partir de l'analyse des articulations entre les blocs pratico-techniques et théorico-technologiques identifiés. Une catégorisation des « savoirs de référence », sur lesquels l'enseignant est susceptible de s'appuyer pour construire ses propres savoirs, a été utilisée. Elle s'inspire des « registres de savoirs » définis par Johsua (1996) ainsi que des distinctions proposées, dans le champ des STAPS, par Léziart (1997). Cinq registres de savoirs appartenant à trois grandes catégories de savoirs sont ainsi spécifiés :

- les «savoirs scientifiques contributoires» et les «savoirs de l'éducation », qui relèvent des «savoirs savants » (liés à des communautés à qui la société a délégué le droit de dire le vrai) ;

- les « savoirs techniques » et les « savoirs didactiques en usage », qui appartiennent à la catégorie des « savoirs experts ${ }^{1}$ (développés par des communautés plus restreintes au sein d'institutions non savantes);

- les « savoirs d'expérience », qui relèvent de « savoirs personnels » propres à un seul individu ou à quelques individus seulement.

C'est donc en analysant les gestes du professeur, que les praxéologies sont reconstruites pour accéder, par inférence, aux savoirs du professeur, eux-mêmes analysés en regard des registres de savoirs.

\section{Méthode}

Nous avons adopté une approche à la fois clinique et expérimentale du didactique ordinaire, dont certains éléments sont empruntés à Leutenegger (2000). Cette méthode d'investigation consiste à orienter l'observation des pratiques ordinaires effectives, à travers un dispositif expérimental adapté aux questions de recherche, tout en rendant compte de la dynamique des systèmes en contexte naturel. Dans notre étude, la

\footnotetext{
${ }^{1}$ Et dans laquelle on peut aussi classer les « savoirs institutionnels ».
} 
dimension clinique est liée au choix d'une étude de cas en situation naturelle et la dimension expérimentale se rapporte à l'introduction du thème d'étude par le chercheur². Le croisement de ces deux approches se double d'une « dialectique entre l'explication et la compréhension des phénomènes en jeu » qui oriente l'organisation progressive des différents corpi (Schubauer-Leoni \& Leutenegger, 2002).

L'analyse porte sur la mise en scène par un enseignant d'EPS, du thème d'étude «franchir par redressement », en saut de cheval, lors des quatre premières séances d'un cycle de gymnastique ${ }^{3}$. Ce thème d'étude renvoie aux "problèmes fondamentaux" de la gymnastique, puisqu'il sollicite les différentes ressources des élèves dans la dialectique "prise de risques - maîtrise de risques" (Goirand, 1987). II représente aussi un thème classique dans l'enseignement scolaire de la gymnastique, étant envisagé dans les programmes d'EPS en cycle d'adaptation et en cycle central (M.E.N, 1996, 1997).

\subsection{L'enseignant retenu pour l'étude}

L'enseignant a été choisi en fonction de son expérience professionnelle. Ce critère repose sur la distinction établie par Elbaz (1981) à partir du nombre d'années de pratique d'enseignement, et rejoint la différenciation plus classique entre experts et novices proposée par les cognitivistes (Tochon, 1991). Dans la perspective anthropologique adoptée ici, l'expérience est vue, avant tout, comme un élément contribuant à définir la trajectoire professionnelle du professeur, notre souci étant de comprendre la spécificité de ses gestes. L'enseignant a aussi été retenu en regard de son niveau d'expertise en gymnastique : non spécialiste de l'activité, il a pratiqué la gymnastique durant sa formation initiale à I'UFRSTAPS. Cet enseignant novice (3 ans d'expérience), appelé Mickaël, a été observé avec une classe de quatrième d'un collège de recrutement «moyen», situé dans l'agglomération d'une petite ville.

2. 2. Le choix des traces et leur transcription

2. 2. 1. Le corpus principal

Le corpus principal est constitué des enregistrements filmés et des entretiens de type « auto-confrontation » menés à l'issue de chaque leçon. Considérant que les savoirs de l'enseignant sont à la fois révélés par ses interactions avec les élèves et par le discours qu'il peut porter sur son activité, nous avons opté pour une méthodologie spécifique couplant: a) une observation des séances ordinaires, telle que réalisée dans les recherches didactiques; b) des entretiens d'autoscopie, appelés « auto-confrontation »,

\footnotetext{
2 Dimension considérée ici comme légère puisque le chercheur ne propose pas de tâches d'apprentissage à l'enseignant.

${ }^{3}$ Cette temporalité caractérise l'entrée dans les apprentissages.
} 
inspirés des méthodes développées en ergonomie et adaptés à notre objet d'étude. Le choix de ces deux outils vise à approcher les différentes formes de savoirs en croisant un point de vue extrinsèque (les actions de l'enseignant filtrées par le regard du didacticien) et un point de vue intrinsèque (le discours de l'enseignant tenu lors des autoconfrontations vidéo avec sa pratique). Un tel couplage méthodologique permet de mettre en relation des données relatives aux trois instances du système didactique.

Ainsi, la transcription simultanée des entretiens d'auto-confrontation et des enregistrements filmés, a permis une identification, en plusieurs temps, des données suivantes :

- des sous-moments de l'étude, terme qui reprend en le spécifiant pour la recherche celui de «moments didactiques » (Chevallard, 2002) et qui permet de repérer l'organisation structurelle et fonctionnelle de la séance ;

- des épisodes, c'est-à-dire des «séries d'interactions relatives au contenu d'enseignement, organisées autour d'une nouvelle tâche » (Amade-Escot, 1996) ;

- des unités d'analyse interactives (UAI) caractérisant l'activité de l'enseignant en réponse à l'activité des élèves, et des unités thématiques (UT), synthétisant le contenu des verbalisations de l'enseignant dans l'entretien.

L'organisation des différents moments de l'étude a mis en évidence les types de tâches proposés par l'enseignant au fil des leçons, et c'est en reprenant chacun des épisodes que nous avons analysé les gestes de conception puis les régulations opérées autour de ces dispositifs.

\section{2. 2. Le corpus secondaire}

Des entretiens de type semi-directif, réalisés en début et en fin de cycle, constituent le corpus secondaire et viennent compléter le corpus principal. L'entretien ante, réalisé avant le cycle, porte sur le contexte d'enseignement et les projets de l'enseignant. II sert à caractériser les éléments contextuels censés influer sur les décisions d'enseignement. L'entretien post, réalisé à l'issue du cycle, est orienté sur les conceptions et savoirs qui guident l'activité de l'enseignant. II vise à formaliser les cadres d'analyse personnels du professeur. Pour ces deux types d'entretiens, un "codage thématique " (Hüberman et Miles, 1991), reprenant chaque verbalisation et s'appuyant sur le guide de questionnement, a été réalisé.

\section{Résultats : des praxéologies reconstruites aux savoirs inférés.}

Nous présenterons, dans un premier temps, les caractéristiques des praxéologies activées par l'enseignant. Celles-ci sont construites à partir d'une analyse de ses gestes 
professionnels. Dans un second temps, nous exposerons les différentes caractéristiques des savoirs qu'il mobilise dans l'action : registres, modalités d'activation, contextes et fonctions des savoirs.

3. 1. L'analyse des gestes professionnels de l'enseignant

Elle a été réalisée en trois temps correspondant aux trois préoccupations suivantes: organiser l'étude, concevoir les dispositifs d'apprentissage et réguler les apprentissages des élèves.

3. 1. 1. Organiser : l'identification des sous-moments consacrés à l'étude

Le cycle de Mickaël repose sur la mise en place de deux ateliers : un atelier sol, où les élèves sont relativement autonomes et un atelier cheval, sur lequel l'enseignant est très présent. Ce choix s'explique par une volonté de gérer la sécurité à l'atelier cheval, considéré comme " dangereux et acrobatique" (entretien ante).

L'analyse structurelle et fonctionnelle de chaque leçon est l'expression des intentions affichées par Mickaël pour conduire son cycle. Elle repose sur l'identification des différents temps consacrés à l'étude, appelés « sous-moments " ${ }^{4}$. Chacun de ces sous-moments se caractérise par des «techniques didactiques» spécifiques, renvoyant aux façons d'intervenir de l'enseignant lorsqu'il dirige l'étude des savoirs (Chevallard, 1999 ; Loquet, 2007). La structure de séance adoptée par l'enseignant est similaire, au cours des différentes séances. Après avoir présenté les objets de travail de la séance à l'ensemble de la classe, un échauffement est proposé. Puis, le troisième sous-moment concerne la "mise en place de la rotation". II a pour fonction de définir les objets de savoir et d'organiser l'engagement des élèves dans la tâche. Le quatrième temps de la séance, consacré aux premiers passages des élèves sur la tâche, vise leur familiarisation avec la tâche demandée. Lors de ce sous-moment, Mickaël utilise des techniques didactiques destinées à rappeler, désigner les objets de savoir ou l'activité attendue, et à commenter, expliquer les réalisations des élèves, à travers les retours verbaux systématiques proposés aux élèves. Le cinquième sous-moment, qui se caractérise par des évolutions du dispositif initial, différenciées selon les rotations, a pour enjeu principal d'installer les élèves dans les apprentissages.

Ces techniques d'intervention révèlent les façons dont l'enseignant dévolue (ou non) aux élèves la responsabilité de leurs apprentissages. Ainsi, l'enseignant prend en charge les interprétations des conduites des élèves, les interventions sur le milieu (changement de

\footnotetext{
${ }^{4}$ Le terme de sous-moment a été introduit pour opérer un découpage plus fin des " moments didactiques ", renvoyant aux fonctions didactiques et aux modalités des différents temps structurant l'étude (Chevallard, 2002).
} 
dispositif, gestion de la distance tremplin-cheval), la différenciation de la tâche en fonction des élèves. II délègue aux élèves la responsabilité de fonctionner seuls, en fin de rotation, ou demande à certains élèves, qu'il désigne, de réaliser la démonstration de la tâche. Les propos recueillis dans les entretiens des séances 2 et 4 révèlent des tensions entre plusieurs types de considération, portant sur la sécurité, l'autonomie, le temps d'action moteur, la motivation, l'investissement et les apprentissages des élèves. Plus généralement, les choix opérés et leur justification dans les entretiens traduisent une forte préoccupation sécuritaire qui explique pour partie le mode d'intervention de Mickaël.

3. 1. 2. Concevoir: l'analyse des différents types de tâches proposées aux élèves

L'organisation des différents sous-moments identifiés au cours de l'analyse précédente s'appuie sur l'élaboration de dispositifs variés qui semblent marquer l'avancée du temps didactique, et dont l'étude plus approfondie nous renseignera sur les connaissances gymniques activées par l'enseignant. Pour chaque dispositif de séance, nous réaliserons une analyse a priori de la tâche, visant à repérer les objets de savoirs en jeu dans la tâche. Puis, en nous appuyant sur les verbalisations de l'enseignant lors des épisodes où il présente la tâche (classées en unités d'analyse interactive) et sur les verbalisations ayant trait à l'analyse des dispositifs lors des entretiens d'auto-confrontation (classées en unités thématiques), nous définirons les savoirs, en jeu dans ces dispositifs pour l'enseignant. La confrontation de ces trois types d'analyse vise à repérer la double logique, gymnique et didactique, sous-jacente à l'articulation de ces différents dispositifs au cours du temps (dimension chronogénétique).

L'étude de ces différents dispositifs confirme l'existence d'une forte relation entre les tâches proposées et la structuration temporelle de l'étude, elle-même dépendante de l'agencement des objets de savoir sous-tendus par la tâche de référence. Le dispositif, conçu autour d'un ou plusieurs objets de savoir déterminés, est en quelque sorte considéré par Mickaël comme un élément déclencheur de l'activité d'apprentissage des élèves. Par exemple, le dispositif «monter - s'asseoir » proposé en séances 3 et 4, vise, pour cet enseignant, à aborder le travail du redressement en en facilitant à la fois les conditions de réalisation et en permettant une moindre appréhension chez les élèves, comme en témoignent à la fois l'analyse des extraits filmés (UAI) où il présente cette tâche aux élèves et des extraits d'entretien d'auto-confrontation s'y rapportant (UT) (tableau 1). 
Tableau I. Extraits des UAI et UT du sous-moment où la tâche est présentée aux élèves, au cours l'épisode 1 de la séance 4.

\begin{tabular}{|c|c|}
\hline $\begin{array}{l}\text { Unités d'analyse } \\
\text { interactive (film) } \\
\text { où Mickaël } \\
\text { présente la tâche }\end{array}$ & $\begin{array}{l}\text { - Premier envol : « avoir la sensation de « je monte et je me laisse } \\
\text { redescendre vers le cheval » } \\
\text { - Redressement : “ repousser dans les épaules », " sans fléchir les } \\
\text { bras », « casser le bas du corps puis se relancer dans les épaules », } \\
\text { "contracter les abdos» }\end{array}$ \\
\hline $\begin{array}{l}\text { Unités } \\
\text { thématiques } \\
\text { (entretiens) tirées } \\
\text { de la séance } 4\end{array}$ & $\begin{array}{l}\text { "J'ai choisi le cheval en mousse, toujours parce que sur le cheval en } \\
\text { dur, ils ont peur et que c'est carrément dangereux... En fait, je l'ai mis } \\
\text { en long pour simplifier la chose, c'est-à-dire que je leur demande de } \\
\text { "pousser dans les épaules et de se redresser en s'asseyant sur le } \\
\text { cheval. Alors comme il est en long, on peut s'asseoir avec une jambe } \\
\text { de chaque côté. C'est plus simple." }\end{array}$ \\
\hline
\end{tabular}

Plus généralement, l'étude de ces différents dispositifs confirme l'existence d'une forte relation entre les tâches proposées et la structuration temporelle de l'étude, elle-même dépendante de l'agencement des objets de savoir sous-tendus par la tâche de référence. En fait, la définition des objets de savoirs repose sur une différenciation globale du premier et du deuxième envol, chacun de ces deux thèmes étant lui-même décomposé en sousthèmes (que nous avons nommés les objets de savoir). Ainsi, le premier envol articule deux objets de savoir : l'impulsion dans le tremplin et l'élévation du bassin de la fin de l'impulsion à la pose des mains. Et le deuxième envol est décomposé en deux objets de savoir : l'appui dynamique des mains sur le cheval et le redressement, qui lui-même se subdivise en deux sous-objets: la fermeture jambes / tronc et la répulsion des bras. L'avancée du temps didactique est ainsi réalisée en respectant les étapes chronologiques du saut, ces dernières étant matérialisées par les différents dispositifs qui se succèdent dans le temps (tableau 2). Si les dispositifs sont centrés sur l'impulsion au cours de la séance 1, ils s'intéressent progressivement à l'élévation du bassin au premier envol, puis, envisagent, en séance 4, le travail du redressement. Pour prendre en compte le rythme d'apprentissage de chacun, Mickaël adapte en fonction des groupes le moment où il introduit tel ou tel dispositif, ce qui ne l'empêche pas de respecter les mêmes étapes. Malgré la volonté de Mickaël d'isoler certains éléments de l'action, la dépendance des différents objets de savoir laisse apparaître des chevauchements, qui témoignent d'une intention d'avancer dans le temps, en partant de l'impulsion sur le tremplin pour aller jusqu'à la réception. 
De manière récurrente, l'enseignant met en scène les objets de savoir, en réduisant la complexité des actions mises en jeu dans la tâche (simplification de la réception pour la tâche «monter - s'asseoir ») et la diminution des contraintes liées à l'aménagement matériel (cheval en mousse placé en long pour la tâche «monter - rouler » ou pour la tâche « monter - s'asseoir »; trampoline pour la tâche « monter - rouler »).

Tableau II. L'identification des objets de savoirs en fonction des dispositifs au cours du cycle de Mickaël.

\begin{tabular}{|c|c|c|c|c|}
\hline Tâche & Descriptif de la tâche & \begin{tabular}{|l|} 
Séance, \\
et \\
rotations $^{5}$
\end{tabular} & $\begin{array}{l}\text { Thème } \\
\text { d'étude } \\
\text { privilégié }\end{array}$ & Objets de savoir \\
\hline $\begin{array}{l}\text { Tâche « sauter - } \\
\text { arriver à genoux } \\
\text { sur le cheval - } \\
\text { saut extension» }\end{array}$ & $\begin{array}{l}\text { - course d'élan } \\
\text { - impulsion } 2 \text { pieds sur le } \\
\text { tremplin } \\
\text { - arriver à genoux sur le } \\
\text { mouton } \\
\text { - se relever puis saut } \\
\text { extension }\end{array}$ & $\begin{array}{l}\text { Séance 1, } \\
\text { en début } \\
\text { de chaque } \\
\text { rotation }\end{array}$ & $1^{\mathrm{er}}$ envol & $\begin{array}{l}\text { Impulsion dans le } \\
\text { tremplin } \\
\text { Appui dynamique } \\
\text { des mains sur le } \\
\text { cheval }\end{array}$ \\
\hline $\begin{array}{l}\text { Tâche } \\
\text { «franchissement } \\
\text { par saut écart » }\end{array}$ & $\begin{array}{l}\text { - course d'élan } \\
\text { - impulsion } 2 \text { pieds sur le } \\
\text { tremplin } \\
\text { - franchissement du mouton } \\
\text { par saut écart } \\
\text { - réception debout sur le } \\
\text { tapis }\end{array}$ & $\begin{array}{l}\text { Séance 1, } \\
\text { en fin de } \\
\text { chaque } \\
\text { rotation } \\
\text { (sauf } \\
\text { rotation 4) }\end{array}$ & \begin{tabular}{|l|} 
Les \\
envols \\
(globalité \\
du saut)
\end{tabular} & $\begin{array}{l}\text { Impulsion dans le } \\
\text { tremplin } \\
\text { Appui dynamique } \\
\text { Redressement : } \\
\text { * Fermeture } \\
\text { active des jambes } \\
{ }^{*} \text { Répulsion bras }\end{array}$ \\
\hline $\begin{array}{l}\text { Tâche « monter - } \\
\text { rouler » avec } \\
\text { trampoline et } \\
\text { cheval mousse } \\
\text { en long }\end{array}$ & $\begin{array}{l}\text { - course d'élan } \\
\text { - impulsion } 2 \text { pieds sur le } \\
\text { trampoline } \\
\text { - élévation du bassin au- } \\
\text { dessus de l'horizontale } \\
\text { avec pose des mains sur le } \\
\text { cheval en mousse placé en } \\
\text { long } \\
\text { - réception en roulade sur le } \\
\text { gros tapis placé en long } \\
\text { derrière le cheval, à même } \\
\text { hauteur }\end{array}$ & $\begin{array}{l}\text { Séance 2, } \\
\text { pour } \\
\text { chaque } \\
\text { rotation et } \\
\text { séance 3 }\end{array}$ & $1^{\mathrm{er}}$ envol & $\begin{array}{l}\text { Elévation au } 1^{\text {er }} \\
\text { envol } \\
\text { Appui dynamique } \\
\text { des mains sur le } \\
\text { cheval }\end{array}$ \\
\hline $\begin{array}{l}\text { Tâche « monter - } \\
\text { s'asseoir " avec } \\
\text { tremplin } r \text { et } \\
\text { cheval mousse } \\
\text { en long }\end{array}$ & $\begin{array}{l}\text { - course d'élan } \\
\text { - impulsion } 2 \text { pieds sur le } \\
\text { tremplin } \\
\text { - élévation du bassin au- } \\
\text { dessus de l'horizontale } \\
\text { avec pose des mains sur le } \\
\text { cheval mousse placé en } \\
\text { long } \\
\text { - réception assise, une } \\
\text { jambe de chaque côté du } \\
\text { cheval en mousse }\end{array}$ & $\begin{array}{l}\text { Séance } 3, \\
\text { pour la fin } \\
\text { de la } \\
\text { rotation } 3 \\
\text { Séance } 4, \\
\text { pour } \\
\text { chaque } \\
\text { rotation }\end{array}$ & \begin{tabular}{|l|}
$2^{\text {ème }}$ envol \\
(phase \\
initiale)
\end{tabular} & $\begin{array}{l}\text { Elévation au } 1^{\text {er }} \\
\text { envol } \\
\text { Appui dynamique } \\
\text { des mains } \\
\text { (blocage épaules) } \\
\text { Fermeture active } \\
\text { des jambes }\end{array}$ \\
\hline
\end{tabular}

${ }^{5}$ Les rotations concernent le changement de groupes sur un même dispositif. Ici, compte- tenu de l'organisation adoptée par Mickaël, il y a 4 rotations. 
3. 1. 3. Réguler : l'analyse des régulations opérées pour chaque dispositif Ce dernier temps de l'analyse s'intéresse aux « gestes d'aide à l'étude » (Chevallard, 1999), qui s'organisent autour des dispositifs conçus par l'enseignant. II concerne l'analyse, pour chaque type de tâche identifié précédemment, des retours verbaux et des régulations sur le milieu didactique. Ce dernier terme désigne le système des objets (matériels, symboliques, langagiers) qui détermine les pratiques d'étude des savoirs (Amade-Escot et al. 2007) et renvoie aux modifications opérées sur le dispositif même. Pour les étudier, nous avons confronté trois types d'analyses: une analyse de l'intervention de l'enseignant dans les sous-moments où il intervient sur la tâche (enregistrement filmé), une analyse des conduites des élèves sur le dispositif concerné6 (enregistrement filmé) et une analyse des interprétations proposées par l'enseignant dans les entretiens d'auto-confrontation.

Comme nous l'avons souligné au cours de l'analyse des sous-moments de l'étude, les régulations verbales - qui désignent les informations que l'enseignant donne aux élèves, avant ou après leurs passages - représentent un mode d'intervention très présent au cours du cycle. Elles sont analysées d'un double point de vue : quantitatif et qualitatif. Les régulations sur le milieu didactique sont spécifiques à un élève donné, ou concernent l'ensemble du groupe. Elles peuvent avoir été anticipées ou non (dans les préparations de séances) et déclenchées ou non (en relation avec certaines conduites repérées par l'enseignant chez les élèves). II s'agira alors d'identifier ce qui déclenche la modification du dispositif et les objets de savoirs envisagés.

Pour Mickaël, les régulations consistent à réaménager le milieu didactique, pour un ou plusieurs élèves, et à produire des retours verbaux systématiques et spécifiques à chaque réalisation d'élève. Les régulations sur le milieu didactique sont différenciées en fonction des réponses des élèves et sont mises en place lorsque les informations qu'il donne verbalement ne produisent pas les transformations attendues. Par exemple, à partir du dispositif «monter - s'asseoir », il met en place plusieurs aménagements du dispositif initial, qui consistent à :

- marquer deux zones d'appui mains sur le cheval ;

- complexifier le franchissement pour les meilleurs élèves, en leur demandant de franchir directement le cheval par saut écart ;

- matérialiser une zone d'impulsion sur le tremplin (ligne tracée à la craie), pour les élèves les plus en difficulté ;

\footnotetext{
${ }^{6}$ Encore appelée, par les didacticiens, analyse a posteriori, et confrontée ici à l'analyse a priori réalisée au moment de l'étude des gestes de conception.
} 
- adapter la distance entre le tremplin et le cheval, en fonction des élèves.

Les régulations sur le milieu didactique consistent, pour l'ensemble des dispositifs conçus (tableau 2), soit à complexifier et/ou simplifier le saut, soit à intervenir sur certains objets de savoirs, en référence à une analyse chronologique du saut et à une forte prise en compte de l'appréhension des élèves.

\section{2. Les savoirs mobilisés par l'enseignant}

L'analyse des fondements théorico-technologiques ${ }^{7}$ impliqués dans la conception et la régulation des dispositifs relatifs au franchissement par redressement au saut de cheval dévoile l'activation de différents types de savoirs de la part de l'enseignant. Pour présenter ces savoirs, nous envisagerons successivement leurs principales caractéristiques: le registre de savoirs auquel elles appartiennent, leurs fonctions et le contexte de leur activation, leurs modalités d'activation.

\section{2. 1. Registres de savoirs}

Les savoirs exploités par Mickaël appartiennent aussi bien aux registres « savants » qu'aux registres « experts» (tableau 3). Ils relèvent du champ de la mécanique, de l'anatomie fonctionnelle, de la psycho-physiologie, du discours didactique gymnique ou de référents didactiques en usage dans la profession. Mickaël s'appuie aussi sur des connaissances issues du champ de la psychologie cognitive et des théories de l'apprentissage moteur pour mettre en relation les caractéristiques psycho-affectives de l'apprenant avec les conditions efficaces d'apprentissage, liées à la réussite, la motivation, l'investissement des élèves, la durée des apprentissages ou au temps d'action moteur. II se réfère aux sciences de l'éducation pour justifier le mode de communication ou le type de pédagogie adopté. Chacun de ces registres de référence, situé dans les catégories élaborées à partir des propositions de Johsua (1996) et de Léziart (1997) ${ }^{8}$ (voir paragraphe 1), est illustré à partir d'un extrait tiré des UAI ou UT relatives aux leçons enregistrées.

La multitude des références expertes et savantes sur lesquelles reposent les gestes de l'enseignant révèle une certaine forme d'incorporation des contenus diffusés en formation initiale, dans le cadre des cursus STAPS, dans la mesure où elles renvoient aux différentes matières qui y sont enseignées. Elle témoigne selon nous d'une activité de recherche importante de la part de Mickaël, qui construit son intervention à travers la convocation et l'agencement spécifique de ces différents éléments. L'actualisation de ces

\footnotetext{
7 Eux-mêmes rattachés aux blocs pratico-techniques.

8 Notons que si ces différentes catégories ont été clairement différenciées, elles se positionnent en réalité sur un continuum.
} 
savoirs dans un contexte donné, exprime l'intégration, l'articulation, la combinaison particulière de ces différents registres de savoirs.

Tableau III. Extraits illustrant les différents registres de savoirs, tirés des UAI ou UT des séances ou des entretiens ante et post.

\begin{tabular}{|c|c|c|c|}
\hline \multicolumn{3}{|c|}{ Registres de savoirs } & Extraits illustratifs \\
\hline \multirow{5}{*}{ 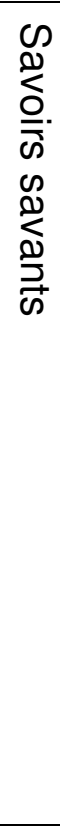 } & \multirow[t]{4}{*}{$\begin{array}{l}\text { Savoirs } \\
\text { scientifiques } \\
\text { contributoires }\end{array}$} & $\begin{array}{l}\text { Mécanique } \\
\text { Anatomie } \\
\text { fonctionnelle }\end{array}$ & $\begin{array}{l}\text { "Le trampolino, il renvoie tellement que ça leur } \\
\text { demande une, euh, une hyperbole vachement } \\
\text { serrée en fait. " (Séance 2, UT23). }\end{array}$ \\
\hline & & $\begin{array}{l}\text { Psycho- } \\
\text { physiologie }\end{array}$ & $\begin{array}{l}\text { «Je leur fais juste faire, [...], une course d'élan avec } \\
\text { taper dans le tremplin pour réadapter les sensations } \\
\text { à l'impulsion [....] II faut qu'ils reprennent leurs } \\
\text { repères par rapport à un tremplin dur, quoi!" } \\
\text { (Séance 2, UT50). }\end{array}$ \\
\hline & & $\begin{array}{l}\text { Théories de } \\
\text { l'apprentissage } \\
\text { (moteur) }\end{array}$ & $\begin{array}{l}\text { "Je leur explique, en jouant peut-être plus sur les } \\
\text { représentations parce que je fais plus de gestes " } \\
\text { (Séance 1, UAl4). }\end{array}$ \\
\hline & & Psychologie & $\begin{array}{l}\text { [...] «leur sentiment d'eux-mêmes, parce qu'il y en a } \\
\text { qui sont pas trop bien dans leur peau dans cette } \\
\text { classe là "... (Entretien ante, US1) }\end{array}$ \\
\hline & $\begin{array}{l}\text { Savoirs de } \\
\text { l'éducation }\end{array}$ & $\begin{array}{l}\text { Sciences de } \\
\text { l'éducation }\end{array}$ & $\begin{array}{l}\text { "C'est uniquement un choix pour travailler le } \\
\text { premier envol, sans leur mettre de contraintes [...]. } \\
\text { C'est un peu, comment dire, décontextualisé en } \\
\text { fait » (séance 2, UT7). }\end{array}$ \\
\hline \multirow[t]{3}{*}{ 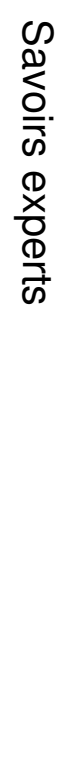 } & \multirow[t]{2}{*}{$\begin{array}{l}\text { Savoirs } \\
\text { didactiques } \\
\text { en usage } \\
\text { dans la } \\
\text { profession }\end{array}$} & $\begin{array}{l}\text { Référents } \\
\text { didactiques } \\
\text { généraux }\end{array}$ & $\begin{array}{l}\text { "Alors, l'an dernier je me disais "Voilà, on doit } \\
\text { partir de l'APS de référence, donc c'est un cheval } \\
\text { en cuir, dur, tanné [...] mais est- ce que l'important } \\
\text { c'est qu'ils passent sur le cheval en dur, où les trois } \\
\text { quarts ont peur [...] ou est-ce que l'important c'est } \\
\text { qu'ils aient une possibilité de varier les sauts? [...] } \\
\text { (Séance 1, UT73). }\end{array}$ \\
\hline & & $\begin{array}{l}\text { Référents } \\
\text { didactiques de } \\
\text { l'activité } \\
\text { gymnique }\end{array}$ & $\begin{array}{l}\text { "J'estime que le cheval est déjà assez } \\
\text { acrobatique [...], mais au sol j'irai plus les chercher } \\
\text { sur "gérer difficultés et propreté " (Entretien ante, } \\
\text { US2). }\end{array}$ \\
\hline & \multicolumn{2}{|c|}{ Savoirs techniques } & $\begin{array}{l}\text { "On pose les mains bras tendus, comme à l'ATR } \\
\text { au sol, on se repousse en faisant une impulsion } \\
\text { dans les épaules, on ramène les jambes et une fois } \\
\text { qu'on est là [mime les trajectoires avec son bras], } \\
\text { on casse le bas du corps et on ramène les jambes, } \\
\text { d'accord? (Séance 3, UAl45) }\end{array}$ \\
\hline
\end{tabular}

3. 2. 2. Fonctions et contextes d'activation des connaissances

Les fonctions assignées à ces savoirs se déclinent autour d'un emboîtement de tâches successives, adaptées et régulées par l'enseignant au fil de l'avancée chronogénétique. Le tableau 4 propose une synthèse articulant « registres de savoir », fonctions et contextes d'utilisation de ces savoirs. 
Tableau IV. Fonctions et contextes d'utilisation des différentes connaissances identifiées.

\begin{tabular}{|c|c|c|}
\hline Registres de savoir & Fonctions des connaissances & Contextes d'utilisation \\
\hline $\begin{array}{l}\text { Mécanique } \\
\text { Anatomie } \\
\text { fonctionnelle } \\
\text { Psycho-physiologie } \\
\text { Techniques }\end{array}$ & $\begin{array}{l}\text { Comprendre et interpréter les } \\
\text { réalisations gymniques des } \\
\text { élèves (en référence à des } \\
\text { critères normés) }\end{array}$ & $\begin{array}{l}\text { Retours verbaux } \\
\text { Conception } \\
\text { dispositifs }\end{array}$ \\
\hline $\begin{array}{l}\text { Théories de } \\
\text { l'apprentissage } \\
\text { moteur, ergonomie } \\
\text { cognitive } \\
\text { Psychologie }\end{array}$ & $\begin{array}{l}\text { Comprendre les mécanismes } \\
\text { impliqués dans les } \\
\text { apprentissages, } \\
\text { comportements des élèves et } \\
\text { adapter les conditions à mettre } \\
\text { en œuvre pour les favoriser }\end{array}$ & $\begin{array}{l}\text { Retours verbaux } \\
\text { Conception } \\
\text { régulation } \\
\text { dispositifs }\end{array}$ \\
\hline $\begin{array}{l}\text { Sciences } \\
\text { l'éducation }\end{array}$ & $\begin{array}{l}\text { Adapter les démarches } \\
\text { d'enseignement aux élèves et à } \\
\text { la spécificité des apprentissages } \\
\text { Adapter le style d'intervention et } \\
\text { des modalités de communication }\end{array}$ & $\begin{array}{l}\text { Conception et gestion } \\
\text { des dispositifs } \\
\text { Retours verbaux }\end{array}$ \\
\hline $\begin{array}{l}\text { Référents didactiques } \\
\text { généraux }\end{array}$ & $\begin{array}{l}\text { Concevoir et adapter les tâches } \\
\text { aux profils des élèves et aux } \\
\text { contenus }\end{array}$ & $\begin{array}{ll}\begin{array}{l}\text { Conception des } \\
\text { dispositifs }\end{array} & \\
\begin{array}{l}\text { Régulations sur le } \\
\text { milieu didactique }\end{array} \\
\end{array}$ \\
\hline $\begin{array}{l}\text { Référents didactiques } \\
\text { de l'activité gymnique }\end{array}$ & $\begin{array}{l}\text { Concevoir, structurer, adapter } \\
\text { les tâches aux profils et aux } \\
\text { difficultés perceptivo-motrices, } \\
\text { affectives des élèves }\end{array}$ & $\begin{array}{l}\text { Conception des } \\
\text { dispositifs } \\
\begin{array}{l}\text { Régulations sur le } \\
\text { milieu didactique }\end{array}\end{array}$ \\
\hline Institutionnels & Justifier les choix réalisés & \\
\hline
\end{tabular}

Au-delà de la multiplicité des savoirs activés par Mickaël, la référence à une option cognitiviste et adaptative des apprentissages, relayée principalement par une analyse technique du saut de cheval et secondairement par des principes d'élaboration des dispositifs, représente le fil conducteur de ses interventions. De façon générale, ces savoirs ont, avant tout, une fonction de guidage des apprentissages.

\section{2. 3. Modalités d'activation des connaissances}

Rappelons qu'au-delà de l'identification de ces savoirs, ce qui nous intéresse particulièrement, c'est la mise à jour des processus de leur activation, parce que nous pensons qu'elle rend possible la généralisation des résultats. La manière dont Mickaël mobilise conjointement plusieurs types de savoirs nous semble relever de processus relativement stables et non pas du hasard de la situation. 
Ainsi, les façons dont Mickaël mobilise ces savoirs in situ sont souvent réalisées en combinant les registres savants et experts, selon des modalités de recoupement qui nous semblent relativement permanentes. L'exploitation de connaissances issues des champs de la mécanique, de l'anatomie fonctionnelle ou de la psycho-physiologie est souvent associée à celle des connaissances techniques. Ainsi, lorsque Mickaël intervient sur la tâche « monter-rouler avec trampoline », il demande à certains élèves de « bien taper au milieu de la toile », "placer les bras tendus, collés aux oreilles », de « gainer ${ }^{9}$, de «garder le corps tendu» pour profiter des «propriétés d'élasticité et de renvoi du trampoline » (séance 2). De même, lorsque l'enseignant se réfère à des savoirs relatifs aux sciences de l'éducation ou aux théories psychologiques, ou encore aux théories de l'apprentissage moteur, il mobilise conjointement des connaissances ayant trait à des référents didactiques en usage dans l'institution. Par exemple, au cours de l'EAC de la séance 2, Mickaël explique le choix de l'utilisation du trampoline par sa dimension motivationnelle en référence au caractère acrobatique de la gymnastique, qui nécessite une certaine « prise de risques».

Si les mises en relation de plusieurs registres obéissent à des principes d'emboîtement relativement stables, elles traduisent plus fondamentalement un glissement entre les registres savants et experts, les derniers rendant les premiers « utilisables » dans la pratique.

\section{Discussion}

\section{1. Registre de savoirs personnels}

Pour caractériser les manières dont les disciplines scolaires mettent en jeu les différentes références, Johsua (1996) emploie l'expression de «mixité des références ». Dans le cadre de notre étude, concernant les savoirs du professeur, cette mixité des références relève d'un processus d'emboîtement, qui traduit des glissements, voire parfois des raccourcis, entre registres experts et savants en fonction des préoccupations inhérentes aux situations pratiques. II nous semble possible d'émettre l'hypothèse selon laquelle ce processus d'opérationnalisation ou de spécification est à l'origine du « registre de savoirs personnels ». Dans le cas de Mickaël, ce registre personnel renvoie à la mise en jeu spécifique et contextualisée des objets de savoirs dans l'aménagement des dispositifs (comme la tâche consistant à monter - rouler par-dessus un élastique tenu par les élèves). II se caractérise aussi par l'articulation spécifique des théories gymniques à dominante

\footnotetext{
${ }^{9}$ Le gainage est un terme introduit par Carrasco qui désigne une posture de placement du bassin en rétroversion associée à une contraction abdominale.
} 
technique (Carrasco, 1972, 1975 ; Piard, 1982) ${ }^{10}$ et à dominante didactique (Goirand, 1990, 1998). Par exemple, la prise en compte de l'appréhension pour concevoir les dispositifs ou l'exploitation de critères articulant les deux phases du saut (position des bras mise en relation avec le redressement) relèvent, chez Mickaël, de principes d'action généralisables, alors que la façon dont il analyse de façon séquentielle les réponses des élèves s'appuie sur la théorie de Carrasco. Plus généralement, les résultats ont révélé que Mickaël tendait à adopter une démarche de plus en plus centrée sur les problèmes des élèves au fur et à mesure qu'il était confronté à leurs réponses sur les dispositifs. De même, s'il valorise une compréhension référée à l'analyse technique du saut dans la conception des dispositifs, il s'appuie davantage, dans ses régulations, sur une interprétation interactive des problèmes des élèves, conformément à la théorie de Goirand. Le registre de savoir personnel concerne aussi les façons dont cet enseignant interprète les conduites gymniques des élèves à partir d'indices particuliers. Par exemple, au cours de la séance 4, dans le cadre des régulations verbales et des interprétations livrées dans les EAC, Mickaël établit une distinction des réponses des élèves à partir de l'observation de la position de la tête par rapport à celle des épaules au moment du contact avec le cheval. Ce registre personnel renvoie aussi à la façon dont l'enseignant construit des références imagées synthétisant des conduites typiques directement communicables aux élèves. C'est le cas lorsqu'il décrit (verbalement et gestuellement) les conduites des élèves lors du premier envol en référence à une posture assise ou debout, au cours de la séance 4.

En fait, la manière dont l'enseignant combine différents registres de savoirs répond au critère d'utilité qui se spécifie dans un processus de glissement entre ces registres. C'est ce mécanisme qui fonde le registre des savoirs personnels.

4. 2. Les savoirs entre convictions personnelles et influences des appartenances institutionnelles

Les savoirs activés par l'enseignant ne sont pas seulement une émanation des registres savants et experts, ils sont aussi l'expression d'interprétations personnelles et contextualisées des situations dans lesquelles ils sont activés.

Tout au long de cette étude, nous avons repéré des tensions entre sécurité et apprentissage, mise en autonomie des élèves - guidage des apprentissages, temps d'engagement moteur - sollicitation de l'activité compréhensive et réflexive des élèves, mises en œuvre pratique et réflexion théorique. A titre d'exemple, les connaissances

\footnotetext{
${ }^{10}$ C'est- à- dire qui définissent les techniques en référence à une norme gestuelle.
} 
activées par Mickaël pour concevoir l'organisation structurelle du cycle (le choix des deux ateliers) et la conception de certaines tâches d'apprentissage (" monter - rouler » en présence d'un trampoline et d'un élastique), sont le résultat de compromis entre des préoccupations sécuritaires et des préoccupations relatives aux apprentissages. C'est à l'occasion de ces conflits que l'activation des connaissances permet à l'enseignant de trouver un compromis acceptable, une solution convenable pour gérer la situation, ici et maintenant. Ainsi, lorsque Mickaël propose le dispositif « monter - rouler » avec trampoline (voir paragraphe 3.3), il tente de dépasser des contradictions entre :

- l'aménagement de conditions sécuritaires et possibilités de renvoi de la surface d'impulsion ${ }^{11}$;

- les intérêts et inconvénients de l'aménagement « cheval en mousse », qui permet une diminution de l'appréhension mais qui pose problème au niveau de l'impulsion des bras, en raison de sa surface molle ;

- la volonté d'isoler le travail du premier envol, et les difficultés de s'appuyer sur un tel travail pour ensuite envisager le travail du redressement (idée de transfert).

Ces diverses tensions sont à l'origine du recours à certains savoirs, issus ici de la mécanique, de la psycho-physiologie, des référents didactiques gymniques précis et secondairement de la psychologie.

Plus généralement, ces tensions dévoilent les convictions propres à l'enseignant ainsi que ses conceptions (relatives à l'enseignement en général, à l'enseignement de l'EPS, à l'enseignement de la gymnastique en milieu scolaire). Mais les savoirs activés in situ dépendent aussi des appartenances institutionnelles du professeur, notamment aux institutions «CAPEPS» et «Ecole ». L'assujettissement à l'Ecole se traduit par les tensions précédemment repérées et par la volonté permanente de faire avancer le temps didactique. Il s'exprime aussi à travers une intériorisation des injonctions officielles, et particulièrement des programmes EPS, comme le montre son attachement aux objectifs suivants: "gérer sa vie physique future », "être en sécurité, s'étirer, s'échauffer », « respecter les autres », «être citoyen» ... (Entretien post-cycle). L'influence de la formation CAPEPS, et notamment de la préparation à la deuxième épreuve écrite de ce concours, qui repose sur la mise en relation de données institutionnelles et scientifiques pour justifier des choix didactiques et pédagogiques, est aussi très prégnante. L'activation des savoirs chez Mickaël relève donc aussi de sa formation initiale, et en particulier de sa

\footnotetext{
${ }^{11}$ Le tremplin renvoyant mieux aux exigences de sécurité et le trampoline favorisant les sensations d'envol et la découverte de la forme du saut.
} 
proximité temporelle avec la préparation au CAPEPS, dans la mesure où elle en reproduit d'une certaine façon la logique. Cet assujettissement à la formation initiale (et notamment à l'institution CAPEPS) s'exprime plus généralement à travers la forme justificative du discours tenu, les choix opérés pour régler les tensions entre plusieurs objectifs apparemment contradictoires, et à travers les contenus, les registres et les modalités de recoupement des différents registres de savoirs activés.

\section{Conclusion}

Cette recherche, à visée descriptive et compréhensive, nous a permis d'explorer, à travers une analyse des pratiques ordinaires, les mécanismes sous-jacents à la mobilisation des connaissances par le professeur d'EPS. Le choix d'une orientation descriptive appelle une autre conception de la preuve, qui repose sur la « production d'une connaissance non pas universelle et définitive (le vrai) mais libérée d'une modélisation limitée au souhaitable », et permettant l'étude des pratiques dans leur diversité et leurs contradictions (Bru, 1998). La mise à jour des processus interactifs développés dans la dynamique des rapports entre enseignement et apprentissage peut alors contribuer à la formulation d'hypothèses relatives aux conditions à mettre en œuvre pour favoriser les apprentissages (Bru, Altet, \& Blanchard-Laville, 2004). Cette recherche a ainsi révélé l'existence de différents processus de mobilisation des savoirs, en particulier d'emboîtement consistant à structurer les mises en relation de ces savoirs ; de recoupement concernant la spécification de ces savoirs en lien avec les pratiques ; et de glissements inter - registres relatifs à l'articulation de différents niveaux de formalisation et d'abstraction des savoirs autour d'un même objet. Comprendre ces mécanismes de combinaison ${ }^{12}$ de savoirs variés peut alors contribuer à concevoir de nouveaux dispositifs de formation initiale, davantage articulés aux pratiques professionnelles. A ce titre, une formation à l'intervention doit permettre à l'étudiant de formaliser, questionner, mettre en relation les différents savoirs scientifiques contributoires dans des contextes proches de leur utilisation, c'est-à-dire en relation avec l'identification des problèmes rencontrés par les élèves dans les diverses APSA.

\section{Bibliographie}

\footnotetext{
12 Par combinaison, nous entendons l'ensemble des processus de mobilisation des savoirs nommés
} précédemment, et concernant leur spécification, leur structuration et l'articulation des divers registres 
Artigue, M. (1988). Ingénierie didactique. Recherche en Didactique des Mathématiques, 9/3, 281-308.

Amade-Escot, C. (1996). L'observation des activités didactiques en Education physique et sportive : aspects méthodologiques. Revue Impulsion, 2, 75-98.

Amade-Escot, C. (1998). Les contenus de l'éducation physique, contribution de deux programmes de recherche. Science et motricité, 32-33, 16-26.

Amade-Escot, C. (2003). La gestion interactive du contrat didactique en volley-ball: agencement des milieux et régulations du professeur. In C. Amade-Escot (Ed.), Didactique de l'éducation physique. Etat des recherches, Paris : Revue EPS, 255278.

Amade-Escot, C. et al. (2007). Le didactique. Paris : Revue EPS, collection « Pour l'action Barbier, J.M. (1996). Savoirs théoriques et savoirs d'action. Paris : PUF.

Brau-Anthony, S. (2001). Les conceptions des enseignants d'EPS sur l'enseignement et l'évaluation des jeux sportifs collectifs : résultats d'une enquête. STAPS, 56, 93108.

Bru, M. (1998). La validation scientifique des propos et discours sur les pratiques d'enseignement: après les illusions perdues. In C.Hadji \& J. Baillé (Eds.), Recherche et éducation. Vers une nouvelle alliance, Bruxelles : De Boeck, 45-65.

Bru, M., Altet, M., \& Blanchard-Laville, C. (2004). A la recherche des processus caractéristiques des pratiques enseignantes dans leurs rapports aux apprentissages. Revue française de pédagogie, 148, 75-87.

Carrasco, R. (1972). Essai de systématique d'enseignement de la gymnastique aux agrès. Paris : Vigot.

Carrasco, R. (1975). L'activité du débutant. Paris : Vigot.

Chevallard, Y. (1989). Le concept de rapport au savoir, rapport personnel, rapport institutionnel, rapport officiel. In Actes du séminaire de didactique des mathématiques et de l'informatique, Grenoble: LSD Imag et Institut J. Fourrier, 211-235.

Chevallard, Y. (1992). Concepts fondamentaux de la didactique : perspectives apportées par une approche anthropologique. Recherche en Didactique des Mathématiques, $12 / 1,73-112$.

Chevallard, Y. (1997). Familière et problématique, la figure du professeur. Recherche en Didactique des Mathématiques, 17/3, 17-54. 
Chevallard, Y. (1999). L'analyse des pratiques enseignantes en théorie anthropologique du didactique. Recherche en Didactique des Mathématiques, 19/2, 221-266.

Chevallard, Y. (2002). Organiser l'étude.1. Structures \& fonctions. In J.L Dorier, M. Artaud, M. Artigue, R. Berthelot, \& Floris (Eds), Actes de la 11e école d'été de didactique des mathématiques -août 2001, La pensée sauvage éditions, 3-22.

Durand, M. (1996). L'enseignement en milieu scolaire. Paris : Presses Universitaires de France.

Elbaz, F. (1981). The teacher's «practical knowledge »: Report of a case study. Curriculum Inquiry, 11, 43-71.

Gauthier, C., \& al. (1997). Pour une théorie de la pédagogie. Recherches contemporaines sur le savoir des enseignants. Québec : De Boeck Université.

Garnier, A. (2003). Le rapport au savoir du professeur : entre contrainte et autonomie. Une étude de cas lors d'un cycle d'enseignement de la gymnastique au collège. Thèse de doctorat en Sciences de l'éducation, non publiée, Université Paul Sabatier, Toulouse III.

Goirand, P. (1987). Des pratiques sociales des APS aux contenus d'enseignement en gymnastique. Spirales, $n^{\circ} 1$, complément, 7-38.

Goirand, P. (1990). Didactique de la gymnastique et éducation physique. In Association des enseignants d'EPS ( Eds.), Education physique et didactique des APS (pp. 74-87). Paris : AEEPS.

Goirand, P. (1998). L'EPS au collège et gymnastique. Paris : INRP.

Hüberman, M., \& Miles, M-B. (1991). Analyse des données qualitatives: recueil de nouvelles méthodes. Bruxelles : De Boeck.

Johsua, S. (1996). Le concept de transposition didactique n'est-il propre qu'aux mathématiques ? In C. Raisky et M.Caillot (Eds), Au-delà des didactiques, le didactique. Débats autour des concepts fédérateurs, Bruxelles : De Boeck, 61-73.

Leutenegger, F. (2000). Construction d'une « clinique » pour le didactique. Une étude des phénomènes temporels de l'enseignement. Recherche en Didactique des Mathématiques, 20/2, 209-250.

Léziart, Y. (1997). Savoirs savants et transposition didactique en éducation physique et sportive. STAPS, 42, 59-72.

Loquet, M. (2007). Les techniques didactiques du professeur. In C.Amade-Escot (coord), Le Didactique (pp. 49-66). Paris : Revue EPS, collection « Pour l'action ».

Margolinas, C. (1992). Eléments pour l'analyse du rôle du maître : les phases de conclusion. Recherche en Didactique des Mathématiques, 12/1, 113-158. 
M.E.N. (1996). Programmes d' EPS de la classe de sixième des collèges. BO $n^{\circ} 29$ du 18 juillet 1996.

M.E.N. (1997). Programmes d’ EPS du cycle central de collège. BO spécial $n^{\circ} 1$ du 13 février 1997.

Mercier, A., Schubauer-Leoni, ML., \& Sensevy, G. (2002). Vers une didactique comparée. Revue française de pédagogie, 141, 5-16.

Piard, C. (1982). Fondements de la gymnastique : technologie et pédagogie. Paris : Vigot.

Polanyi, M. (1967). The tacit dimension. New-York : Doubleday.

Perrin-Glorian, M.J. (1993). Questions didactiques soulevées à partir de l'enseignement des mathématiques dans les « classes faibles». Recherche en Didactique des Mathématiques, 13/1-2, 5-118.

Schubauer-Leoni, M.L., \& Leutenegger F. (2002). Expliquer et comprendre dans une approche clinique/expérimentale du didactique ordinaire. In F. Leutenegger, \& M. Saada-Robert (Eds), Expliquer et comprendre en sciences de l'éducation (pp. 227-251). Paris : Bruxelles : De Boeck.

Robin, J.F. (1998). Spécificité, structure et sens de savoirs pour enseigner en gymnastique scolaire: étude de 4 leaders de théories didactiques. Thèse de doctorat STAPS, mention didactique des APS, non publiée, Université de Paris XI, Orsay.

Tochon, F. (1991). Les critères d'expertise dans la recherche sur les enseignants. Mesure et évaluation en éducation, 14(2), 57-81.

Vandevelde, M. (2001). L'action de formation d'Alès en basket-ball : étude de cas Contribution à l'analyse du système didactique de formation continue en EPS. Thèse de doctorat en sciences de l'éducation, non publiée, Université Paul Sabatier, Toulouse III. 